Polymer Journal, Vol. 38, No. 7, pp. 639-642 (2006)

(C) 2006 The Society of Polymer Science, Japan

\title{
Non-Polar Polymer Nanotubes and Nanowires Fabricated by Wetting Anodic Aluminium Oxide Template
}

\author{
Xilin SHE, Guojun Song, ${ }^{\dagger}$ Jianjiang LI, Ping Han, Shujing YANG, \\ Wang SHulONG, and Zhi PENG
}

Institute of Polymer Materials, Qingdao University, Qingdao, 266071 China

(Received January 13, 2006; Accepted February 20, 2006; Published May 29, 2006)

\begin{abstract}
Non-polar polymer nanotubes and nanowires were fabricated using nanoporous Anodic Aluminium Oxide (AAO) template via a physical wetting of the polymer solution and melt. Testing results of field emission scanning electron microscopy (FESEM) demonstrate that non-polar polymer nanotubes can be prepared by polymer solution, while nanotubes and nanowires can be obtained by polymer melt at higher temperature and at lower temperature respectively. As for melt wetting method, there is a critical temperature for preparing nanotubes, and the structure of products depends on the viscosity and flowability of the polymer melt. The mechanism of melt wetting method is also discussed. [doi:10.1295/polymj.PJ2005208]

KEY WORDS Non-Polar Polymer / Nanotubes / AAO / Wetting Template Method /
\end{abstract}

In recent years, one-dimension (1D) polymer nanomaterials, such as nanotubes, nanowires and nanocables, have attracted considerable attention because of their outstanding functions and special applications. Especially, polymer nanotubes has attracted growing research interest due to their novel properties and wide potential application for nanodevices. ${ }^{1-7}$

There are two main methods for preparing polymer nanotubes in AAO template. One is chemical synthesis; the other is physical wetting. Many polar polymer nanotubes have been reported so far. Most of them have been prepared using former method, such as Polyamide 6, Poly-L-lactide (PLLA), Polytetrafluoroethylene (PTFE), Poly (vinylidene difluoride) (PVDF), and so on, ${ }^{2,4-6,8}$ and some of them have been prepared by the latter one. ${ }^{3,9-11}$ However, the preparation of non-polar polymer nanotubes has rarely been reported.

Herein, we developed an effective method for preparing non-polar polymer nanotubes by polymer solution (or melt) wetting AAO template, and successfully prepared two typical non-polymer nanotubes and nanowires with normal molecule weight polypropylene (PP) and low-density polyethylene (LDPE). Their morphology was characterized by FESEM. A new viewpoint on mechanism was put forward for preparing non-polar polymer nanotubes by the wetting method.

\section{EXPERIMENTAL}

\section{Preparation of PP Nanotubes}

Anodic aluminum oxide (AAO) with $200 \mathrm{~nm}$ diameter pores was purchased from Whatman International
Ltd. Prior to using it; AAO was stirred by ultrasonic wave to clean its surface. PP was solved into decahydronaphthalene to prepare $5.0 \mathrm{wt} \% \mathrm{PP}$ solution at $120^{\circ} \mathrm{C}$.

Polymer Solution Wetting Method. A drop of solution was placed on a microscope slide and then a commercial AAO membrane was quickly placed on it. The solution entered completely into the template pores along their inner wall in $2 \mathrm{~s}$. And the solvent also completely evaporated after $1-2 \mathrm{~min}$. Above experimental was operated in an oven at $60-80^{\circ} \mathrm{C}$ in order to insure the molecules of PP did not separate out from the solution. Then, the AAO/PP composite membrane was removed from the microscope slide by soaking the assembly in hot water, and then placed in the $3 \mathrm{M}$ sodium hydroxide solution in order to dissolve the template. PP nanotubes were obtained.

Polymer Melt Wetting Method. A platelet of PP was heated to $200{ }^{\circ} \mathrm{C}$ or $250^{\circ} \mathrm{C}$ on a hot plate to melt it. A round AAO membrane was then placed on top of the polymer melt and maintained at the temperature for several minutes. The PP melt entered into the pores of the membrane due to capillary force and adhesive forces between the surface of template and the polymer melt. After about $5 \mathrm{~min}$, the hot plate was allowed to slowly cool to room temperature, and then the PP composite membrane was removed from the glass slide by soaking the assembly in hot water. The composite membrane was placed in the $3 \mathrm{M}$ sodium hydroxide solution in order to dissolve the template. PP nanotubes were obtained. Same, LDPE nanotubes and nanowires were, respectively, prepared at $160^{\circ} \mathrm{C}$ and $200^{\circ} \mathrm{C}$ by this method.

\footnotetext{
${ }^{\dagger}$ To whom correspondence should be addressed (E-mail: songguojun@qdenc.com).
} 


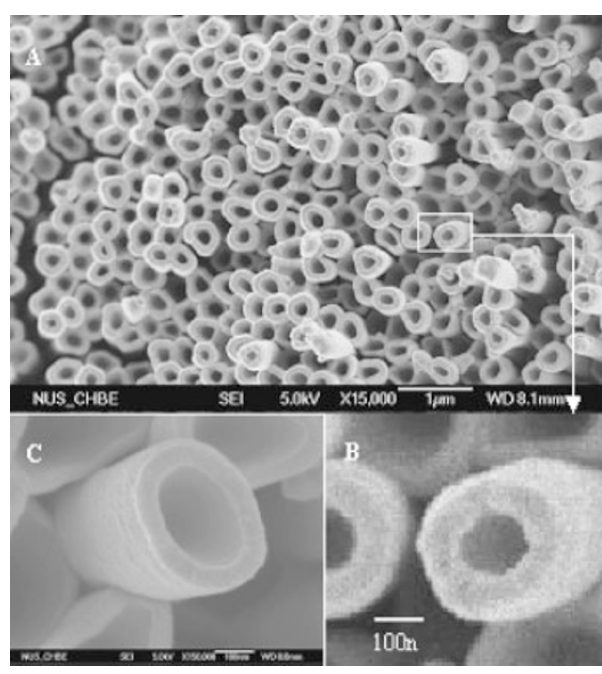

Figure 1. FESEM images of polymer nanotubes prepared from $5.0 \mathrm{wt} \%$ solution. (A) PP nanotubes prepared from polymer solution at $60^{\circ} \mathrm{C}$; (B) the magnified image of a select region in Figure 1A; (C) PA66 nanotubes prepared from $5.0 \mathrm{wt} \%$ polymer solution at room temperature.

\section{Morphology of Nanotubes}

Field Emission Scanning Electron Microscopy (FESEM) The alumina membrane with PP nanotubes was fixed on a microscope slide and placed in the $3 \mathrm{M}$ sodium hydroxide solution to dissolve the alumina. The surface of the sample was then sputtered with $\mathrm{Au}$ and imaged with a JEOL FESEM-6700 or JEOL JSM-840 scanning electron microscope.

\section{RESULTS AND DISCUSSION}

\section{Morphology and Structure of PP Nanotubes and Nanowires}

Figure 1A shows the FESEM image of PP nanotubes prepared by $5.0 \mathrm{wt} \%$ PP solution wetting method. From it we can see that the PP nanotubes with regular array structure have formed but they arrange closely after removing AAO template. Figure 1B depicts the magnified image of a select region in Figure 1A. It can be found the nanotubes have about
$300 \mathrm{~nm}$ outer diameter, a $100 \mathrm{~nm}$ wall thickness and a very plain mouth.

Figure 1C presents polyamide 66 (PA66) nanotubes prepared from $5.0 \mathrm{wt} \%$ polymer solution. Comparing Figure 1B with Figure 1C, it can clearly be seen that the wall thickness of PP nanotubes $(\sim 100 \mathrm{~nm})$ is larger than that of PA66 nanotubes $(\sim 60 \mathrm{~nm})$ prepared from the same concentration solution. The reasons may be as follows. Firstly, the actual concentration of PP solution might increase because of the solvent volatilizing at the high temperature $\left(\sim 80^{\circ} \mathrm{C}\right)$ in an oven. Secondly, the concentration of polymer solution might seriously affect the wall thickness of nanotubes. The higher the concentration of the solution, the thicker the wall of the nanotubes is. ${ }^{12,13}$ For the same weight percent concentration, the volume percent concentration of PP solution will be higher than that of PA66 solution because the density of PA66 $(1.15 \mathrm{~g} /$ $\left.\mathrm{cm}^{3}\right)$ is obviously higher than that of PP $(0.91 \mathrm{~g} /$ $\mathrm{cm}^{3}$ ). So PP nanotubes have the thicker wall than PA66 nanotubes.

Figure 2 presents the SEM images of PP nanowires and nanotubes prepared by melt-wetting method. Figure 2A shows the morphology of the one-dimension nanostructures obtained at $200{ }^{\circ} \mathrm{C}$. There are nanowires instead of nanotubes. Each nanowire has a depression on the top end. So it can be speculated that the nanotubes are just the precursor of nanowires, i.e., nanowires derive from nanotubes.

Figure 2B shows the PP nanotubes prepared at $250^{\circ} \mathrm{C}$. They have a $300 \mathrm{~nm}$ outer diameter and a $80 \mathrm{~nm}$ wall thickness, and their mouths are not very plain and the wall thickness is not uniform. Comparing Figure 2B with Figure 1B, the wall of nanotubes from melt is apparently thinner than that of nanotubes prepared with polymer solution.

The main reason why nanotubes can't be obtained at $200^{\circ} \mathrm{C}$ is that the viscosity of PP melt at $200^{\circ} \mathrm{C}$ is higher than at $250^{\circ} \mathrm{C}$. For a certain template and a polymer melt, there is a constant driving force for the melt to climb along the inner wall of pores. For a certain polymer, the higher the viscosity, the higher

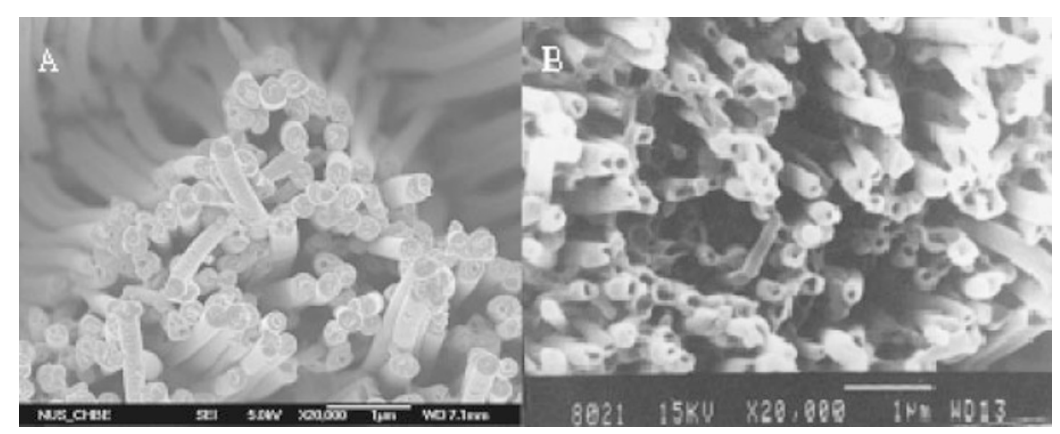

Figure 2. SEM images of PP nanowires and nanotubes from PP melt at $200^{\circ} \mathrm{C}(\mathrm{A})$ and $250^{\circ} \mathrm{C}(\mathrm{B})$. 

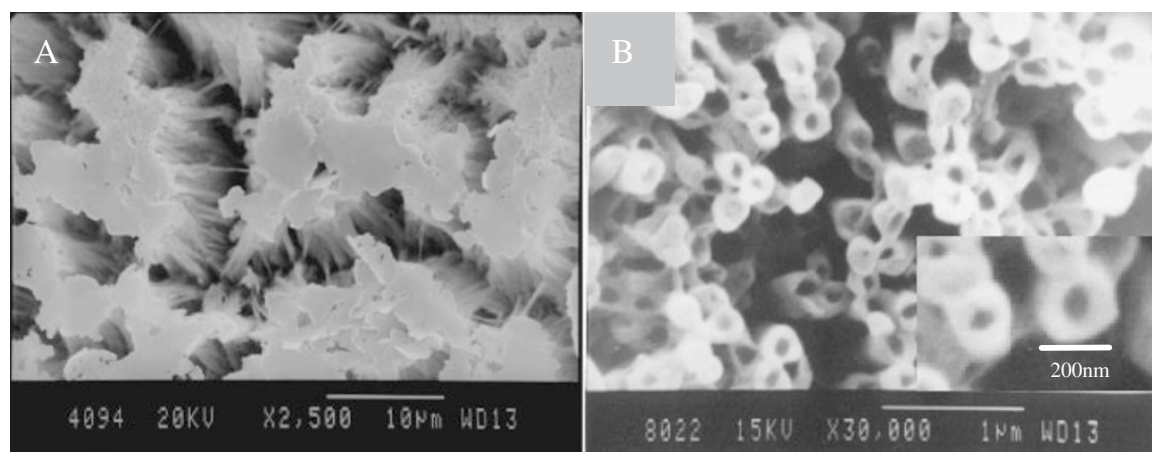

Figure 3. SEM images of LDPE nanotubes prepared by melt wetting at $160^{\circ} \mathrm{C}(\mathrm{A})$ and $200^{\circ} \mathrm{C}(\mathrm{B})$.

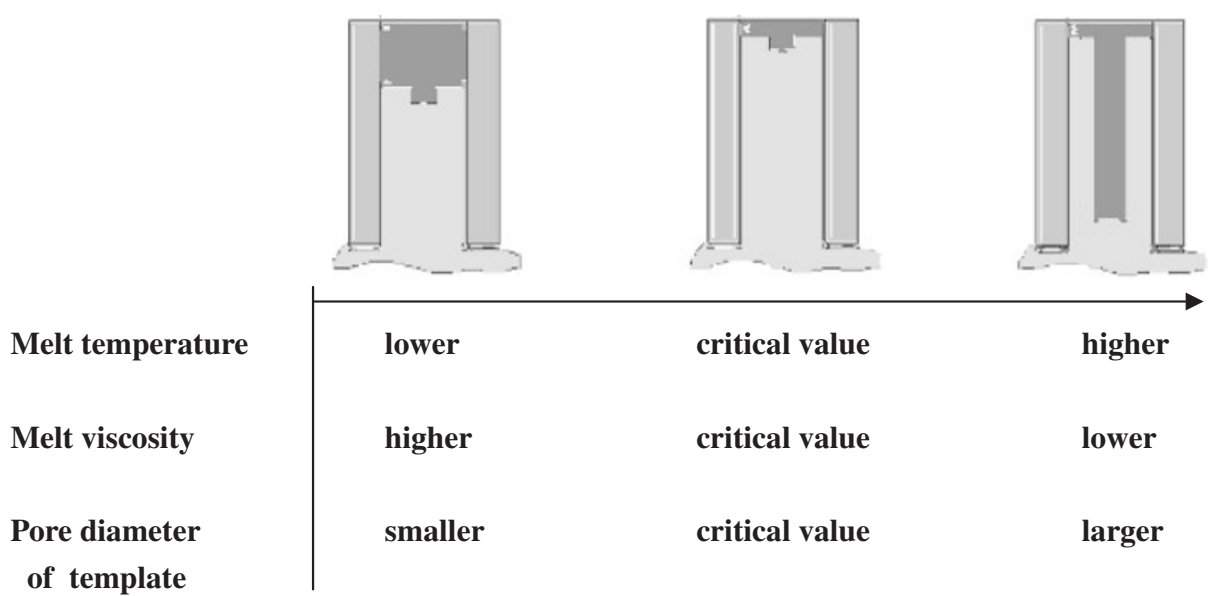

Figure 4. Schematic diagram of the critical value mechanism of wetting AAO template by polymer melt.

the cohesive force of melt is. Under the higher cohesive force, more melt enters into pores and then forms a thicker polymer layer on the inner wall of template pores. If the thickness of the wetting layer is just equal to the radius of nanopores, PP nanowires will be prepared; otherwise, PP nanotubes will be obtained. So there must be a critical temperature value between $200^{\circ} \mathrm{C}$ and $250^{\circ} \mathrm{C}$ for preparing PP nanotubes by melt-wetting method.

\section{Morphology of LDPE Nanotubes and Nanowires}

Figure 3 depicts low-density polyethylene (LDPE) nanowires and nanotubes fabricated by melt-wetting method at different temperature. Similar to PP, LDPE nanowires were also obtained at the relative low temperature $\left(160^{\circ} \mathrm{C}\right)$ shown in Figure $3 \mathrm{~A}$, and nanotubes were achieved at the relative high temperature $\left(200^{\circ} \mathrm{C}\right)$ shown in Figure 3B. These nanotubes have about $300 \mathrm{~nm}$ outer diameter and $70 \mathrm{~nm}$ wall thickness.

Critical Value Principle for Preparing Any Polymer Nanotubes by Melt-wetting Method

As to PP, Figure 2 depicts polymer nanowires formed at $200{ }^{\circ} \mathrm{C}$ (shown in Figure 2A) and polymer nanotubes formed at $250^{\circ} \mathrm{C}$ (shown in Figure 2B). Figure 3 shows LDPE nanowires were also obtained at the relative low temperature $\left(160^{\circ} \mathrm{C}\right)$, and nanotubes were achieved at the relative high temperature $\left(200^{\circ} \mathrm{C}\right)$. It indicates that the experimental temperature is a key factor in preparing polymer nanotubes by melt-wetting method. Obviously, there is a critical temperature value for forming nanotubes between $200^{\circ} \mathrm{C}$ and $250^{\circ} \mathrm{C}$. When the experimental temperature is lower than the critical temperature, we shall prepare polymer nanowires, whereas, polymer nanotubes will be obtained at higher temperature. Figure 4 shows the forming mechanism of one-dimension nanostructures.

Combining with the research results of Greiner and McCarthy, we speculate on the mechanism of forming nanotubes by polymer melt-wetting method. Because of a very high surface energy on the inner surface of template nanopores, the adhesion force between polymeric melt and nanoporous wall is much bigger than the cohesion force of molecule chains in polymer melt. After entering into nanopores, polymeric melt first wets nanoporous wall and forms a wetting layer by the adhesion force. The wetting layer extends along porous wall to the other template surface. If 
the thickness of wetting layer is less than the radius of nanopores, polymer nanotubes will be obtained, otherwise, polymer nanowires will be prepared.

The wetting layer thickness depends on the viscosity of polymeric melt. The lower melt temperature, the higher the viscosity of melt is, and a thicker wetting layer will be formed. Surely, a polymeric melt possesses a certain viscosity at a certain temperature, and there should be a condition under which the thickness of wetting layer is just equal to the radius of nanopores. The temperature and viscosity of the condition are the critical values for corresponding polymeric melt. Furthermore, for a polymeric melt possessing a certain temperature and a certain viscosity, the structure of one-dimension materials will lie on the diameter of template pores. Namely, to prepare polymer nanotubes, there must be a critical value for the diameter of template's pores.

\section{Mechanism of Preparing Non-polar Polymer Nano- tubes by Melt-wetting Method}

Some reports indicate the inner wall of AAO template pores possesses a strong polarity. ${ }^{14-16}$ According to the traditional wetting principle, polar polymer melt can easily wet the polar wall of template pores. It provides enough driving force to form $1 \mathrm{D}$ polymer nanostructures in AAO nanopores by a wetting method. However, it is almost impossible for a non-polar polymer melt to wet a normal polar wall of template pores, because there is an energy barrier between these two materials. Only if the energy barrier is overcome, non-polar 1D structures will be fabricated.

There is just a high surface energy on the inner surface of AAO nanopores. When the polymer melt touches the high energetic surface, most of the surface energy will be transformed into the combining energy. The combining energy is high enough to overcome the energy barrier and provides a driving force to wet the porous walls and make polymer melt rise along the inner wall.

According to above discussion and our experimental results, it is concluded that the polarity of polymer melt has hardly influences on preparing $1 \mathrm{D}$ polymer nanostructrues and there is high surface energy in inner wall of nanoporous template. So the classical wetting principal is not suitable for preparing polymer nanostructures in the AAO template. This mechanism of preparing $1 \mathrm{D}$ polymer nanomaterials by the solution method is similar to the melt method and is ver- satile for any polymer. The research has promising significance for preparing non-polar polymer nanotubes by template method.

\section{CONCLUSION}

Non-polar polymer (PP and LDPE) nanotubes and nanowires are obtained in AAO template nanopores by solution-wetting method and melt-wetting method. As for melt-wetting method, there is a critical temperature for preparing nanotubes. The driving force of wetting template arises from the high surface energy of the template inner wall and is high enough to overcome the energy barrier between non-polar polymer melt and a polar alumina surface for preparing the polymer one-dimension nanomaterials.

Acknowledgment. This work was financially supported by the National Nature Science Foundation of China (NNSFC 50473012).

\section{REFERENCES}

1. C. R. Martin, Science, 266, 1961 (1994).

2. S. Stewart and G. J. Liu, Angew. Chem., Int. Ed., 39, 340 (2000).

3. M. Steinhart, J. H. Wendorff, and A. Greiner, Science, 296, 1997 (2002).

4. M. Steinhart, S. Senz, and R. B. Wehrspohn, Macromolecules, 36, 3646 (2003).

5. Z. M. Zhang and M. X. Wan, Synth. Met., 128, 83 (2002).

6. H. Q. Cao, Z. Xu, and D. Sheng, Adv. Mater., 13, 121 (2001).

7. G. K. Mor, K. Shankar, M. Paulose, O. K. Varghese, and C. A. Grimes, Nano Lett., 5, 191 (2005).

8. V. M. Cepak and C. R. Martin, Chem. Mater., 11, 1363 (1999).

9. K. Huang and M. X. Wan, Chem. Mater., 14, 3486 (2002).

10. S. I. Moon and T. J. McCarthy, Macromolecules, 36, 4253 (2003).

11. S. F. Ai, G. Lu, Q. He, and J. B. Li, J. Am. Chem. Soc., 125, 11140 (2003).

12. G. J. Song, X. L. She, Z. F. Fu, and J. J. Li, J. Mater. Res., 19, 3324 (2004).

13. G. J. Song, X. L. She, and J. J. Li, J. Qingdao Univ., 19, 25 (2004).

14. L. S. Van Dyke and C. R. Martin, Langmuir, 6, 1118 (1990).

15. W. Liang and C. R. Martin, J. Am. Chem. Soc., 112, 9666 (1990).

16. J. C. Bao and Z. Xu, Chin. J. Inorg. Chem., 18, 965 (2002). 